\title{
The State of Kidney Transplantation in the United States
}

\author{
Robert Wolfe \\ Department of Biostatistics, University of Michigan, Ann Arbor, Michigan
}

\begin{abstract}
Although transplantation is the preferred treatment for end-stage renal disease (ESRD) patients compared to dialysis, the limited number of organ donors limits this treatment option for many patients. Various approaches to increase the number of donors are currently being investigated. While the quality of life and
\end{abstract}

patient survival are far from optimal for ESRD patients, improvements have been seen recently in both dialysis and transplantation outcomes. Prevention of ESRD is preferred to either treatment option.
The state of kidney transplantation in the United States only makes sense when discussed in the context of the overall pattern of treatment for end-stage renal disease (ESRD). The U.S. Renal Data System (USRDS) and the Scientific Registry of Transplant Recipients (SRTR) are national data sources for ESRD and transplant data, respectively, and many of the statistics reported here are from those registries.

There were more than 100,000 new ESRD patients reported in the United States in 2002, and recently this count has been rising by about $2-4 \%$ per year (1). The recent growth in incidence counts is primarily due to increases in the size of the U.S. population rather than to increases in the incidence rate per million population. While the incidence rates increased by about $6 \%$ annually between 1990 and 1996, they have become more stable since 1999, with less than $1 \%$ growth per year, on average. ESRD incidence rates decreased between 1999 and 2002 for patients less than 65 years old, but the rates continue to increase for older patients. Even if incidence rates stabilize, the financial costs of providing care for ESRD patients can be projected to grow based on the growth and aging of the U.S. population. Current efforts to prevent ESRD and its precursor, chronic kidney disease (CKD), may lead to effective strategies to reduce the incidence of or delay the onset of ESRD in the United States. Until such strategies are found, the medical costs of treating the growing ESRD population will continue to mushroom.

Seventy-two percent of U.S. ESRD patients are treated by dialysis, with $91 \%$ of them receiving in-center hemodialysis and the remainder receiving peritoneal dialysis or another home treatment. The remaining $28 \%$

Address correspondence to: Robert Wolfe, PhD, Department of Biostatistics, University of Michigan, 109 South Observatory St., Ann Arbor, Ml 48109, or e-mail: bobwolfe@umich.edu.

Seminars in Dialysis—Vol 18, No 6 (November-December) 2005 pp. 453-455 of ESRD patients, more than 120,000 people, have functioning kidney transplants.

Compared to dialysis, transplantation has consistently been found to be a superior renal replacement therapy with regard to quality of life (2-4). Evans et al. (2) reported better levels of functional status, ability to work, and subjective measures of quality of life among transplant recipients, after adjusting for patient characteristics.

It is now widely believed that in addition to improving the quality of life, transplantation also extends the patient's life. Therefore, for many ESRD patients, transplantation is now the preferred treatment option. After the early postoperative period, in which the relative risk of death is greater among transplanted patients than among patients on dialysis, there is an overall survival benefit for transplanted patients that is seen within 8 months after transplantation. Following successful transplantation, patients have a $25-50 \%$ longer projected lifetime compared to patients remaining on dialysis (5). In that study, lower death rates were found in all patient groups studied, including elderly (older than age 60 years) and diabetic recipients. However, a recent study has found no survival benefit from transplantation among morbidly obese patients (6).

There has been no large-scale randomized trial comparing transplantation and dialysis, so it is difficult to know how much of the transplant benefit might be due to selection of healthier patients for transplantation compared to those remaining on dialysis, but substantial effort has been devoted to controlling for factors affecting outcomes in comparative survival analyses (7). When comparing dialysis and transplant outcomes, it is important both to adjust for patient characteristics and to select dialysis controls from among wait-listed patients, who tend to be healthier on average than the wider population of all dialysis patients.

While transplantation is a successful alternative to dialysis for ESRD patients, there can be adverse outcomes for some transplant recipients, including perioperative mortality and graft rejection. The need for continued 
immunosuppression therapy also has an adverse impact on quality of life for many transplant recipients. Nevertheless, there has been steady improvement in the outcome of renal transplantation, due in part to new immunosuppressive agents and a better understanding of how to use them. Between 1993 and 1998, the 1-year graft failure rate for deceased donor kidney transplants declined from $18 \%$ to $11 \%$ and graft failure outcomes have been stable since then $(8,9)$. One-year patient survival rates have been greater than $94 \%$ throughout the same period and 5 -year patient survival is now greater than $80 \%$.

Although current evidence indicates that following renal transplantation better quality of life and longer lifetimes can be expected for nearly all major groups of patients, dialysis remains the predominant therapy for ESRD patients. Why? Unfortunately, the number of transplantable organs has not kept up with the number of ESRD patients seeking transplantation (8). The number of deceased donor renal transplants in the United States remained relatively flat, at about 7700 between 1994 and 1997. By 2003 the number had grown to 8389 , a $2.3 \%$ increase over the number in 2002 . Over the same period, the number of living donor renal transplants grew from 3007 in 1994 to 6464 in 2003. Including kidney-pancreas transplants, there were 14,853 kidney transplants in the United States in 2003, compared to the 100,000 new ESRD patients and 23,488 new additions to the kidney transplant wait-list in 2002. This disparity has been occurring for many years and in 2003 there were 57,211 candidates awaiting kidney transplantation in the United States. The number of new wait-listed kidney registrants less than age 50 years has remained fairly stable since 1994 , but the number of new registrants age 50-64 years has doubled, and the number of new registrants older than age 64 years has more than tripled during the past decade.

Efforts are currently under way to increase the number of deceased donor kidneys available for transplantation by procuring organs from a greater percentage of eligible donors (currently about $45 \%$ of potential eligible donors become actual donors), by increasing the number of kidneys recovered from each donor, by widening the criteria of donor acceptability, and by increasing the utilization of organs (approximately 11\% of recovered organs are not used, mostly due to the quality of the organ) $(8,9)$. The transplant community often focuses on the size of the kidney transplant wait-list as a measure of the shortage of organs, but one could speculate that if more donor organs become available, there may be many more dialysis patients who opt to be put on the transplant waiting list.

Graft failure and posttransplant patient survival rates vary greatly, depending upon the characteristics of the donor, especially donor age, but also upon a history of hypertension, elevated creatinine, and cause of death (10). Donors with certain combinations of these characteristics have been labeled expanded criteria donors (ECD). There is wide variation in the utilization rates of ECD organs that are considered for transplantation. While the utilization rate for recovered deceased donor kidneys is about $89 \%$ overall, it is closer to $50 \%$ for kidneys recovered from donors older than age 60 years. Recent changes have been made to the waiting list system for ECD kidneys in order to streamline the use of
ECD kidneys by explicitly identifying candidates who are willing to accept organs from these higher risk donors. Although these ECD kidneys have approximately $70 \%$ higher failure rates than non-ECD organs, they are still associated with lower death rates and longer lifetimes compared to remaining on dialysis (11). However, recent SRTR studies of recipients of ECD kidneys in Pennsylvania (12) showed that hospitalization rates remained higher through the first 24 months after transplantation than they were before transplantation, suggesting that the quality of life benefit seen for transplants in general needs further investigation among ECD transplant recipients.

What impact does transplantation have upon individual dialysis centers? Selected transplant statistics are now reported to dialysis facilities in their ESRD network reports, including the number and percentage of their patients who have been transplanted or placed on the transplant waiting list. However, there are many steps in the process of getting a kidney transplant and the most important role for dialysis facilities might well be patient education about treatment options.

One question often asked by dialysis centers is "How would higher transplant rates affect the mortality results among the patients who remain on dialysis at my facility?" While no one answer will fit all dialysis facilities, analyses of facility statistics have found only a very weak relationship between transplantation rates and standardized mortality rates. It appears that although healthier patients are more likely to be transplanted, much of that effect of patient selection is accounted for by the adjustments made to mortality statistics among those remaining on dialysis.

Patients often ask about their prospects for a transplant if they are put on the waiting list. The answer varies greatly according to where the patient lives, insurance status, age, blood type, degree of sensitization [panel reactive antibodies (PRA)], and the human leukocyte antigen (HLA) phenotype of the patient. Geography plays the largest role, with more than threefold differences among regions. Some candidates try to reduce their waiting times by registering in multiple regions. Transplant outcomes are better for patients who have had less exposure to dialysis, so earlier listing and transplantation is clearly advantageous.

Although the number of ESRD patients continues to climb and the number of deceased donor transplants has remained relatively flat in recent years, the prognosis of individual ESRD patients has improved in many ways. Dialysis outcomes have improved dramatically in recent years, with mortality rates decreasing among dialysis patients by approximately 5\% (13), perhaps because of improvements in the adequacy of dialysis and anemia management (core indicators study). These improvements in dialysis care have likely contributed to longer dialysis lifetimes and are also likely to have improved quality of life for these patients (especially the improvements in anemia management). Although important, these improvements fall short of the benefits seen from transplantation. Continued efforts to improve immunosuppression therapy, to increase the number of donor organs available, and to make the allocation process more efficient and equitable are likely to lead to better transplant outcomes as well. 
Despite the focus in this article on the glass half full due to improved treatment of ESRD, as a faculty member in a school of public health, I must end with the maxim that prevention of disease is better than the treatment of disease. All of the efforts to improve the treatment of ESRD would pale in importance in comparison to substantial progress in reducing the incidence of ESRD.

\section{References}

1. U.S. Renal Data System: USRDS 2002 Annual Data Report: Atlas of EndStage Renal Disease in the United States. Bethesda, MD: National Institutes of Health, National Institute of Diabetes and Digestive and Kidney Diseases, 2002

2. Evans RW, Manninen DL, Garrison LP, Hart LG, Blagg CR, Gutman RA, Hull AR, Lowrie EG: The quality of life of patients with end-stage renal disease. N Engl J Med 312:553-559, 1985

3. Johnson JP, McCauley CR, Copley JB: The quality of life of hemodialysis and transplant patients. Kidney Int 22:826-291, 1982

4. Simmons RG, Anderson C, Kamstra L: Comparison of quality of life of patients on continuous ambulatory peritoneal dialysis, hemodialysis and after transplantation. Am J Kidney Dis 4:253-255, 1984

5. Wolfe RA, Ashby VB, Milford EL, Ojo AO, Ettenger RE, Agodoa LYC, Held PJ, Port FK: Comparison of mortality in all patients on dialysis, patients on dialysis awaiting transplantation, and recipients of a first cadaveric transplant. N Engl J Med 341:1725-1730, 1999

6. Pelletier SJ, Maraschio MA, Schaubel DE, Dykstra DM, Punch JD, Wolfe RA, Port FK, Merion RM: Survival benefit of kidney and liver transplantation for obese patients on the waiting list. In Terasaki PI, Cecka JM, (eds). Clinical Transplants. Los Angeles: UCLA Tissue Typing Laboratory, 2003:77-88

7. Schaubel DE, Dykstra DM, Murray S, Ashby VB, McCullough KP, Dickinson DM, Hulbert-Shearon TE, Webb RL, Wolfe RA: Analytical approaches for transplant research. Am J Transplant 4(4 pt 2):950-957, 2005

8. U.S. Organ Procurement and Transplantation Network and the Scientific Registry of Transplant Recipients: Transplant Statistics: 2004 Annual Report. Rockville, MD: Department of Health and Human Services, Health Resources and Services Administration, Healthcare Systems Bureau, Division of Transplantation, 2004

9. Port FK, Dykstra DM, Merion RM, Wolfe RA: Organ donation and transplantation trends in the USA, 2003. Am J Transplant 4(suppl 9):7-12, 2004

10. Metzger RA, Delmonico FL, Feng S, Port FK, Wynn JJ, Merion RM: Expanded criteria donors for kidney transplantation. Am J Transplant 3(suppl 4):114-125, 2003

11. Ojo AO, Hanson JA, Meier-Kriesche HU, Okechukwu CN, Wolfe RA, Leichtman AB, Agodoa LY, Kaplan B, Port FK: Survival in recipients of marginal cadaveric donor kidneys compared with other recipients and waitlisted transplant candidates. J Am Soc Nephrol 12:589-597, 2001

12. Wolfe RA, Dykstra DM, Leichtman AB, Port FK: Hospitalization and death rates for Pennsylvania kidney transplant recipients by donor type and HLA mismatch. Am J Transplant 5(suppl 11):A851, 2005

13. Wolfe RA, Hulbert-Shearon TE, Ashby VB, Mahadevan S, Port FK: Improvements in dialysis patient mortality are associated with improvements in urea reduction ratio and hematocrit, 1999-2002. Am J Kidney Dis 45:127-135, 2005 